Commun. Korean Math. Soc. 21 (2006), No. 1, pp. 165-175

\title{
RIBAUCOUR TRANSFORMATIONS OF THE SURFACES WITH CONSTANT POSITIVE GAUSSIAN CURVATURES IN THE 3-DIMENSIONAL EUCLIDEAN SPACE
}

\author{
JOONSANG PARK
}

\begin{abstract}
We associate the surfaces of constant Gaussian curvature $K=1$ with no umbilics to a subclass of the solutions of $O(4,1) / O(3) \times O(1,1)$-system. From this correspondence, we can construct new $K=1$ surfaces from a known $K=1$ surface by using a kind of dressing actions on the solutions of this system.
\end{abstract}

\section{Introduction}

Recently, the theory of surfaces has been studied extensively in differential geometry. Classically, many mathematicians had interests in the surfaces in the 3-dimensional Euclidean space $\mathbb{R}^{3}$ which have special geometric properties that they have good coordinate systems so that the corresponding Gauss-Codazzi equations have nice forms and they have geometric transformations to construct such surfaces from known ones. The well-known examples are surfaces with constant Gaussian curvatures, surfaces with constant mean curvatures and so on, and there are transformations for such surfaces studied by Bianchi, Bäcklund, Ribaucour and Darboux, etc. Nowadays, the Gauss equations of such surfaces are known as soliton equations and those transformations can be explained as the loop group actions on the solution spaces of the corresponding equations.

Received October 13, 2005.

2000 Mathematics Subject Classification: 57N35.

Key words and phrases: Gaussian curvature, sinh-Gordon equation, $G / K$-system, flat connection, sphere congruence, Ribaucour transformation.

This work was supported partially by Dongguk University and by Korea Institute for Advanced Study. 
A surface with the Gaussian curvature $K=1$ which has no umbilic points is characterized by the so-called sinh-Gordon equation

$$
\Delta \phi+\sinh \phi \cosh \phi=0,
$$

which turns out to be a soliton equation.

$L$. Bianchi was the first one who found a geometric method to construct a new $K=1$ surface from a known $K=1$ surface [1]. The so-called Bianchi-Bäcklund transformation is obtained by taking line congruences twice. Recently, U. Hetrich-Jeromin and F. Pedit [5], using quaternionic calculation, showed that Bianchi- Bäcklund transformations are Darboux transformations for isothermic surfaces, and F. Burstall [3] showed that Darboux transformations are equivalent to some loop group actions of the simple type on the quaternion algebra. Finally, S. Kobayashi and J. Inoguchi [4] proved that all the above three transformations are equivalent for $K=1$ or $H=1$ surface.

In this paper, we introduce the $O(4,1) / O(3) \times O(1,1)$-system, which is a $G / K$-system defined by C. L. Terng [8], and we relate a subclass of the solutions of this system to the $K=1$ surfaces with no umbilics. Also, we construct Ribaucour transformations on these surfaces, which are sphere congruences, using some actions on the solutions of this system.

\section{Surfaces in $\mathbb{R}^{3}$ with positive constant Gaussian curvature}

In this section, we investigate on the characterization of the surface in $\mathbb{R}^{3}$ with $K=1$ which has no umbilic points.

Let $X: M \longrightarrow \mathbb{R}^{3}$ be an immersed surface. Take a local orthonormal frame $e_{1}, e_{2}, e_{3}$ on $M$ so that $e_{3}$ is normal to $M$. Let $\omega_{1}$ and $\omega_{2}$ be the dual coframe field on $M$. For the local invariants of $M$, we use the following convention. Let $\omega_{i j}$ be the connection 1-form, that is,

$$
d e_{i}=\sum_{i=1}^{3} e_{j} \otimes \omega_{j i} .
$$

The shape operator $A$ is

$$
A=d e_{3}=e_{1} \otimes \omega_{13}+e_{2} \otimes \omega_{23},
$$

and the first and the second fundamental forms are

$$
\begin{aligned}
& I=\omega_{1} \otimes \omega_{1}+\omega_{2} \otimes \omega_{2}, \\
& I=\omega_{1} \otimes \omega_{13}+\omega_{2} \otimes \omega_{23} .
\end{aligned}
$$


The structure equations, the Gauss and the Codazzi equations are

$$
\begin{aligned}
& d \omega_{1}+\omega_{2} \wedge \omega_{21}=0, \quad d \omega_{2}+\omega_{1} \wedge \omega_{12}=0, \\
& d \omega_{12}=K \omega_{1} \wedge \omega_{2}=\omega_{13} \wedge \omega_{23}, \\
& d \omega_{13}=-\omega_{12} \wedge \omega_{23}, \quad d \omega_{23}=-\omega_{21} \wedge \omega_{13} .
\end{aligned}
$$

The $K=1$ surface with no umbilics is characterized by the sinhGordon equation

$$
\Delta \phi+\sinh \phi \cosh \phi=0 .
$$

The following theorem for the $K=1$ surfaces is well-known, to which we give an elementary proof below.

Theorem 2.1. Suppose $X: M \longrightarrow \mathbb{R}^{3}$ is an immersed surface which has the Gaussian curvature $K=1$ with no umbilic points. Then there exists an isothermal and line of curvature coordinate system $(x, y)$ on $M$ and a function $\phi$ such that

$$
\left\{\begin{array}{l}
I=\cosh ^{2} \phi d x^{2}+\sinh ^{2} \phi d y^{2} \\
I I=\sinh \phi \cosh \phi\left(d x^{2}+d y^{2}\right)
\end{array}\right.
$$

Moreover, the Gauss equation for $M$ is

$$
\Delta \phi+\sinh \phi \cosh \phi=0 .
$$

Conversely, if $\phi$ is a solution of (2.4), then there exists a $K=1$ surface in $\mathbb{R}^{3}$ whose fundamental forms are given as above.

Proof. Take a local orthonormal frame $e_{1}, e_{2}, e_{3}$ on $M$ so that

$$
A\left(e_{1}\right)=\tanh \phi e_{1}, \quad A\left(e_{2}\right)=\operatorname{coth} \phi e_{2},
$$

where $\tanh \phi$ and $\operatorname{coth} \phi$ are the principal curvatures and $K=\tanh \phi$ $\operatorname{coth} \phi=1$.

By the Frobenius theorem, there exists $(u, v)$ such that $e_{1}=\frac{1}{E} \frac{\partial}{\partial u}$ and $e_{2}=\frac{1}{G} \frac{\partial}{\partial v}$ for some $E$ and $G$. So $\omega_{1}=E d u$ and $\omega_{2}=G d v$.

By the structure equations (2.1) and from (2.5), we have

$$
\omega_{12}=\frac{E_{v}}{G} d u-\frac{G_{u}}{E} d v
$$




$$
\omega_{13}=\tanh \phi \omega_{1}=E \tanh \phi d u,
$$$$
\omega_{23}=\operatorname{coth} \phi \omega_{2}=G \operatorname{coth} \phi d v
$$

Substituting (2.6), (2.7) and (2.8) to the Codazzi equations (2.3), we obtain

$$
\begin{aligned}
& E_{v}(\tanh \phi-\operatorname{coth} \phi)+E \phi_{v} \operatorname{sech}^{2} \phi=0, \\
& G_{u}(\tanh \phi-\operatorname{coth} \phi)+G \phi_{u} \operatorname{csch}^{2} \phi=0 .
\end{aligned}
$$

This implies that

$$
(E \operatorname{sech} \phi)_{v}=0,(G \cosh \phi)_{u}=0 .
$$

Hence, there exist functions $a(u)$ and $b(v)$ such that

$$
E \operatorname{sech} \phi=a(u), \quad G \cosh \phi=b(v) .
$$

Choose $x$ and $y$ such that $d x=a(u) d u$ and $d y=b(v) d v$. Then

$$
\begin{aligned}
\omega_{1} & =E d u=\cosh \phi d x, \quad \omega_{2}=G d v=\sinh \phi d y, \\
\omega_{13} & =\tanh \phi \omega_{1}=\sinh \phi d x, \quad \omega_{23}=\operatorname{coth} \phi \omega_{2}=\cosh \phi d y .
\end{aligned}
$$

Therefore,

$$
\left\{\begin{array}{l}
I=\cosh ^{2} \phi d x^{2}+\sinh ^{2} \phi d y^{2}, \\
I I=\sinh \phi \cosh \phi\left(d x^{2}+d y^{2}\right) .
\end{array}\right.
$$

On the other hand, by differentiating (2.9), we obtain $\omega_{12}=\phi_{y} d x-\phi_{x} d y$. Thus by the Gauss equation (2.2), we have $d \omega_{12}=-\Delta \phi d x \wedge d y=$ $1 \cdot \omega_{1} \wedge \omega_{2}$ so that $\Delta \phi+\sinh \phi \cosh \phi=0$.

Conversely, suppose $\phi$ is a solution of the sinh-Gordon equation (2.4). It follows from the fundamental theorem of surface theory that there exists a surface $M$ such that $I$ and $I I$ are as above and it is easy to check that $M$ has $K=1$.

3. $O(4,1) / O(3) \times O(1,1)$-system and $K=1$ surfaces

Let $G / K$ be a symmetric space . $G / K$-system was defined by C. L. Terng [8], which is very useful in the study of submanifold geometry. In particular, when $G / K$ is a Grassmannian manifold, $G / K$-system is 
related with some nice submanifolds in the space forms [2], [6], [7]. Here, we briefly introduce the $O(4,1) / O(3) \times O(1,1)$-system whose details are referred to [2], and explain how this system is related to the $K=1$ surfaces.

Denote by $\mathcal{M}_{m \times n}$ the set of $m \times n$ matrices, $\mathcal{O}$ a simply connected open subset in $\mathbb{R}^{3}$ containing $(0,0), \delta=\operatorname{diag}(d x, d y) \in \mathcal{M}_{2 \times 2}, J=$ $\operatorname{diag}(1,-1) \in \mathcal{M}_{2 \times 2}$ and let $g l_{*}(n)=\left\{F \in \mathcal{M}_{n \times n} \mid f_{i i}=0\right.$ for any $\left.i\right\}$.

Definition 3.1. ([2]) The $O(4,1) / O(3) \times O(1,1)$-system is a partial differential equation for $(F, G): \mathcal{O} \subset \mathbb{R}^{2} \longrightarrow g l_{*}(2) \times \mathcal{M}_{1 \times 2}$ such that

$$
\theta_{\lambda}=\left(\begin{array}{ccc}
\delta F^{t}-F \delta & \delta G^{t} & -\lambda \delta J \\
-G \delta & 0 & 0 \\
\lambda \delta & 0 & \delta F-J F^{t} \delta J
\end{array}\right)
$$

is a family of flat connections on $\mathcal{O} \subset \mathbb{R}^{2}$ for all $\lambda \in \mathbb{C}$. The map $E$ such that $E^{-1} d E=\theta_{\lambda}$ and $E(0,0, \lambda)=0$ is called a frame for the solution $(F, G)$.

Here, the matrix $\theta_{\lambda}$ is partitioned into blocks so that

$$
\omega=\left(\begin{array}{cc}
\delta F^{t}-F \delta & \delta G^{t} \\
-G \delta & 0
\end{array}\right) \in o(3), \quad \eta=\delta F-J F^{t} \delta J \in o(1,1) .
$$

Since $\theta_{\lambda}$ in (3.1) is flat for any $\lambda$, so are $\omega$ and $\eta$. Thus there exist maps $A: \mathcal{O} \longrightarrow O(3)$ and $B: \mathcal{O} \longrightarrow O(1,1)$ such that

$$
A^{-1} d A=\omega, \quad B^{-1} d B=\eta .
$$

Since $B$ is $O(1,1)$-valued, we may assume

$$
B=\left(\begin{array}{cc}
\cosh \phi & \sinh \phi \\
\sinh \phi & \cosh \phi
\end{array}\right)
$$

Then by (3.3), we have

$$
\eta=\left(\begin{array}{cc}
0 & d \phi \\
d \phi & 0
\end{array}\right)=\left(\begin{array}{cc}
0 & f_{12} d x+f_{21} d y \\
f_{12} d x+f_{21} d y & 0
\end{array}\right)
$$

and thus $f_{12}=\phi_{x}$ and $f_{21}=\phi_{y}$. Hence we say $\left(\phi, g_{1}, g_{2}\right)$ is a solution or not instead of $(F, G)$ being so, where $G=\left(g_{1}, g_{2}\right)$.

Taking a gauge transformation on $\theta_{\lambda}$ by $h=\left(\begin{array}{cc}A & 0 \\ 0 & B\end{array}\right)$, we obtain a flat connection

$$
h * \theta_{\lambda}=\left(\begin{array}{cc}
0 & -\lambda A_{1} \delta B^{t} J \\
\lambda B \delta A_{1}^{t} & 0
\end{array}\right)
$$

where $A=\left(A_{1}, A_{2}\right)=\mathcal{M}_{3 \times 2} \times \mathcal{M}_{3 \times 1}$. 
Proposition $3.2([2])$. Suppose $\left(\phi, g_{1}, g_{2}\right)$ is a solution of the $O(4,1)$ $/ O(3) \times O(1,1)$-system and let $\theta_{\lambda}$ be the corresponding flat connection. Then

(i)

$$
\left\{\begin{array}{l}
\Delta \phi+g_{1} g_{2}=0 \\
\left(g_{1}\right)_{y}=\phi_{y} g_{2} \\
\left(g_{2}\right)_{x}=\phi_{x} g_{1} .
\end{array}\right.
$$

(ii) There exists a map $X=\left(X_{1}, X_{2}\right): \mathcal{O} \longrightarrow \mathcal{M}_{3 \times 2}$ such that $d X=A_{1} \delta B^{t} J$, where $A$ and $B$ are given by (3.3),

(iii) $X$ can be obtained by the Sym's formula

$$
\left.\frac{\partial E}{\partial \lambda} \cdot E^{-1}\right|_{\lambda=0}=\left(\begin{array}{cc}
0 & -X \\
J X^{t} & 0
\end{array}\right),
$$

where $E$ is the frame for $\theta_{\lambda}$, that is, $E^{-1} d E=\theta_{\lambda}$ and $E(0,0, \lambda)=0$. (iv) Put $A=\left(e_{1}, e_{2}, e_{3}\right) \in \mathbb{R}^{3} \times \mathbb{R}^{3} \times \mathbb{R}^{3}$. Then $e_{1}, e_{2}, e_{3}$ are a local orthonormal frame for $X_{1}$ and $X_{2}$, and

$$
\left\{\begin{array}{l}
d X_{1}=e_{1} \otimes \cosh \phi d x+e_{2} \otimes \sinh \phi d y, \\
d X_{2}=-\left(e_{1} \otimes \sinh \phi d x+e_{2} \otimes \cosh \phi d y\right), \\
d e_{3}=e_{1} \otimes g_{1} d x+e_{2} \otimes g_{2} d y
\end{array}\right.
$$

(v) $X_{1}$ and $X_{2}$ have the same Gaussian curvatures

$$
K_{1}=K_{2}=\frac{g_{1} g_{2}}{\sinh \phi \cosh \phi} .
$$

Now, we explain the relation between nonumbilic $K=1$ surfaces and solutions of the $O(4,1) / O(3) \times O(1,1)$-system.

THEOREM 3.3. $\phi$ is a solution of the sine-Gordon equation (2.4) if and only if $(\phi, \sinh \phi, \cosh \phi)$ is a solution of the $O(4,1) / O(3) \times O(1,1)$ system. In this case, the immersion $X_{1}$ in the Proposition 3.2 is a $K=1$ surface with no umbilics such that the fundamental forms are

$$
\left\{\begin{array}{l}
I_{1}=\cosh ^{2} \phi d x^{2}+\sinh ^{2} \phi d y^{2} \\
I_{1}=\sinh \phi \cosh \phi\left(d x^{2}+d y^{2}\right) .
\end{array}\right.
$$

Proof. Substitute $g_{1}=\sinh \phi$ and $g_{2}=\cosh \phi$, then the result follows from the Proposition 3.2 by a direct calculation.

REMARK 3.4. In the above theorem, $X_{2}$ becomes a totally umbilic surface in $\mathbb{R}^{3}$. 


\section{Transformations}

Now, we construct an action on the solutions of the form $(\phi, \sinh \phi$, $\cosh \phi)$ of the $O(4,1) / O(3) \times O(1,1)$-system $(c f$. [2]).

Consider the bilinear form $<,>_{1}$ on $\mathbb{C}^{5}$ given by

$$
<u, v>_{1}=\sum_{i=1}^{4} \overline{u_{i}} v_{i}-\overline{u_{5}} v_{5} .
$$

Let $W=\left(w_{1}, w_{2}, w_{3}\right)^{t}$ and $Z=\left(z_{1}, z_{2}\right)^{t}$ be unit vectors in $\mathbb{R}^{3}$ and the Lorentzian space $\mathbb{R}^{1,1}$, respectively, and let $\pi$ be the orthogonal projection of $=\mathbb{C}^{5}$ onto $\mathbb{C}\left(\begin{array}{c}W \\ i Z\end{array}\right)$ with respect to $<,>_{1}$. So, $\pi=$

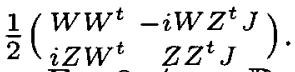

For $0 \neq s \in \mathbb{R}$, define

$$
q_{s, \pi}(\lambda)=\left(\pi+\frac{\lambda-i s}{\lambda+i s}(I-\pi)\right)\left(\bar{\pi}+\frac{\lambda+i s}{\lambda-i s}(I-\bar{\pi})\right) .
$$

Let $(F, G)$ be the solution of the $O(4,1) / O(3) \times O(1,1)$-system, $\theta_{\lambda}$ the corresponding flat connection as in (3.1), and $E$ be the frame of $(F, G)$, i.,e., $E^{-1} d E=\theta_{\lambda}$ and $E(0,0, \lambda)=0$.

Put $\left(\begin{array}{c}\widetilde{W} \\ i \widetilde{Z}\end{array}\right)(x, y)=E(x, y,-i s)^{-1}\left(\begin{array}{c}W \\ i Z\end{array}\right)$ and let $\widetilde{\pi}(x, y)$ be the orthogonal projection onto $\mathbb{C}\left(\begin{array}{l}\widetilde{W} \\ i \widetilde{Z}\end{array}\right)$. Denote by

$$
\widetilde{E}(x, y, \lambda)=E(x, y, \lambda) q_{s, \widetilde{\pi}}(\lambda)^{-1}, \quad \widehat{W}=\frac{\widetilde{W}}{\|\widetilde{W}\|_{3}}, \quad \widehat{Z}=\frac{\widetilde{Z}}{\|\widetilde{Z}\|_{1,1}} .
$$

LEMMA 4.1. The followings hold [2].

(i) $\widetilde{\theta}_{\lambda}=\widetilde{E}^{-1} d \widetilde{E}$ gives a new solution $(\widetilde{F}, \widetilde{G})$ of the $O(4,1) / O(3) \times$ $O(1,1)$-system,

(ii)

$$
\begin{aligned}
& E(x, y, 0)=\left(\begin{array}{cc}
A(x, y) & 0 \\
0 & B(x, y)
\end{array}\right), \\
& \widetilde{E}(x, y, 0)=\left(\begin{array}{cc}
\widetilde{A}(x, y) & 0 \\
0 & \widetilde{B}(x, y)
\end{array}\right),
\end{aligned}
$$

for some $A, \widetilde{A} \in O(3)$ and $B, \widetilde{B} \in O(1,1)$, and

$$
\widetilde{A}=A\left(I-2 \widehat{W} \widehat{W}^{t}\right), \quad \widetilde{B}=B\left(I-2 \widehat{Z} \widehat{Z}^{t} J\right) .
$$


(iii)

$$
\left(\begin{array}{c}
\widetilde{F} \\
\widetilde{G}
\end{array}\right)=\left(\begin{array}{c}
F \\
G
\end{array}\right)-2 s\left(\widehat{W} \widehat{Z}^{t} J\right)_{*}
$$

where $\left(c_{i j}\right)_{*}$ means $c_{i i}=0$.

(iv) $(\widetilde{W}, \widetilde{Z})$ is a solution of

$$
d\left(\begin{array}{l}
\widetilde{W} \\
i \widetilde{Z}
\end{array}\right)=-\theta_{-i s}\left(\begin{array}{l}
\widetilde{W} \\
i \widetilde{Z}
\end{array}\right)
$$

(v) $X, \widetilde{X}: \mathcal{O} \longrightarrow \mathcal{M}_{3 \times 2}$ given by

$$
\left.\frac{\partial E}{\partial \lambda} \cdot E^{-1}\right|_{\lambda=0}=\left(\begin{array}{cc}
0 & -X \\
J X^{t} & 0
\end{array}\right) \text { and }\left.\frac{\partial \widetilde{E}}{\partial \lambda} \cdot \widetilde{E}^{-1}\right|_{\lambda=0}==\left(\begin{array}{cc}
0 & -\widetilde{X} \\
J \widetilde{X}^{t} & 0
\end{array}\right)
$$

satisfy

$$
\widetilde{X}=X-\frac{2}{s} A \widehat{W} \widehat{Z}^{t} B^{t} J
$$

This action by $q_{s, \pi}(\lambda)$ of the form (4.1) on the immersions $X=$ $\left(X_{1}, X_{2}\right)$ can be interpreted as a geometric transformation.

Definition 4.2 . Let $M$ and $\widetilde{M}$ be surfaces in $\mathbb{R}^{3}$.

(1) A sphere congruence is a diffeomorphism $l: M \longrightarrow \widetilde{M}$ such that the lines normal to $M$ at $p$ and $\widetilde{M}$ at $l(p)$ intersect at a point equidistant to $p$ and $l(p)$.

(2) A sphere congruence $l: M \longrightarrow \widetilde{M}$ is called a Ribaucour transformation if any principal vector $e_{p}$ to $M$ is sent to a principal vector $l_{*}\left(e_{p}\right)$ to $\widetilde{M}$ and the lines in these directions at $p$ and $l(p)$ intersect at a point equidistant to $p$ and $l(p)$.

Proposition 4.3. Let $X=\left(X_{1}, X_{2}\right)$ and $\widetilde{X}=\left(\widetilde{X}_{1}, \widetilde{X}_{2}\right)$ be given by (v) in the Lemma 4.1. Then $X_{i}$ and $\widetilde{X}_{i}$ are in a Ribaucour transformation. In particular,

$$
X_{j}+\psi_{i j} e_{i}=\widetilde{X}_{j}+\psi_{i j} \widetilde{e}_{i},
$$

where $\psi_{i j}=-\frac{1}{s \hat{w}_{i}}\left(\widehat{Z}^{t} B^{t} J\right)_{1 j}$ and $\widetilde{A}=\left(\widetilde{e}_{1}, \widetilde{e}_{2}, \widetilde{e}_{3}\right)$. 
Proof. The result follows from (4.2) and (4.5).

Now, we will show that the action by $q_{s, \pi}(\lambda)$ on the solution $(\phi, \sinh \phi$, $\cosh \phi)$ of the $O(4,1) / O(3) \times O(1,1)$-system gives a new solution of the same form.

Theorem 4.4. Suppose $(\phi, \sinh \phi, \cosh \phi)$ is a solution corresponding to $\theta_{\lambda}$ in (3.1). Let $X=\left(X_{1}, X_{2}\right)$ be given as in the Lemma $4.1(\mathrm{v})$. Let $q_{s, \pi}(\lambda), E, W, Z, \widetilde{E}, \widetilde{W}, \widetilde{Z}, \widetilde{\theta}_{\lambda}, \widetilde{X}$ be defined as in the Lemma 4.1.

If $s w_{3}=z_{1} \sinh \phi(0,0)+z_{2} \cosh \phi(0,0)$, then $(\widetilde{\phi}, \sinh \widetilde{\phi}, \cosh \widetilde{\phi})$ is a solution corresponding to $\widetilde{\theta}_{\lambda}$, where $\widetilde{\phi}=2 \alpha-\phi$ and $\left(\widehat{z}_{1}, \widehat{z}_{2}\right)=(\cosh \alpha$, $-\sinh \alpha)$. In this case,

$$
\widetilde{X}_{1}=X_{1}-\frac{2}{s}\left(\widehat{z}_{1} \cosh \phi+\widehat{z}_{2} \sinh \phi\right) \sum_{i=1}^{3} \widehat{w}_{i} e_{i}
$$

and the immersions $X_{1}$ and $\widetilde{X}_{1}$ are $K=1$ surfaces with no umbilics and they are in a Ribaucour transformation.

Proof. From (4.4),

$$
\left\{\begin{array}{l}
d \widetilde{w}_{1}=-\widetilde{w}_{2} \omega_{12}-\widetilde{w}_{3} \sinh \phi d x+s \widetilde{z}_{1} d x \\
d \widetilde{w}_{2}=-\widetilde{w}_{1} \omega_{21}-\widetilde{w}_{3} \cosh \phi d y-s \widetilde{z}_{2} d y \\
d \widetilde{w}_{3}=\widetilde{w}_{1} \sinh \phi d x+\widetilde{w}_{2} \cosh \phi d y \\
d \widetilde{z}_{1}=s \widetilde{w}_{1} d x-\widetilde{z}_{2} d \phi \\
d \widetilde{z}_{2}=s \widetilde{w}_{2} d y-\widetilde{z}_{1} d \phi .
\end{array}\right.
$$

Thus $d\left(s \widetilde{w}_{3}-\widetilde{z}_{1} \sinh \phi-\widetilde{z}_{2} \cosh \phi\right)=0$ and thus by the initial condition,

$$
s \widetilde{w}_{3}=\sinh \phi \widetilde{z}_{1}+\cosh \phi \widetilde{z}_{2},
$$

or, $s \widehat{w}_{3}=\sinh \phi \widehat{z}_{1}+\cosh \phi \widehat{z}_{2}=\sinh (\phi-\alpha)$, where $\left(\widehat{z}_{1}, \widehat{z}_{2}\right)=(\cosh \alpha$, $-\sinh \alpha)$. Hence by (4.3),

$$
\left\{\begin{array}{l}
\widetilde{g}_{1}=\sinh \phi-2 s \widehat{w}_{3} \widehat{z}_{1}=\sinh \phi-2 \sinh (\phi-\alpha) \cosh \alpha=\sinh \widetilde{\phi}, \\
\widetilde{g}_{2}=\cosh \phi+2 s \widehat{w}_{3} \widehat{z}_{2}=\cosh \phi-2 \sinh (\phi-\alpha) \sinh \alpha=\cosh \widetilde{\phi},
\end{array}\right.
$$

and thus $(\widetilde{\phi}, \sinh \widetilde{\phi}, \cosh \widetilde{\phi})$ is a new solution. Therefore $X_{1}$ and $\widetilde{X}_{1}$ are nonumbilic $K=1$ surfaces. From (4.2), we have

$$
\begin{aligned}
\widetilde{B} & =B\left(I-\widehat{Z} \widehat{Z}^{t} J\right) \\
& =\left(\begin{array}{cc}
-\cosh (2 \alpha-\phi) & -\sinh (2 \alpha-\phi) \\
\sinh (2 \alpha-\phi) & \cosh (2 \alpha-\phi)
\end{array}\right)=\left(\begin{array}{cc}
-\cosh \widetilde{\phi} & -\sinh \widetilde{\phi} \\
\sinh \widetilde{\phi} & \cosh \widetilde{\phi}
\end{array}\right),
\end{aligned}
$$


and thus $\widetilde{\phi}=2 \alpha-\phi$. The formula for $\widetilde{X}_{1}$ comes from (4.5) and the fact that $X_{1}$ and $\widetilde{X}_{1}$ are in a Ribaucour transformation follows from the Proposition 4.3.

EXAMPLE 4.5. $\phi=0$ is the trivial solution of the sinh-Gordon equation. By acting $q_{s, \pi}(\lambda)$ on $\phi=0$ and for $s=\sinh c$, we have

$$
\left(\begin{array}{c}
\widetilde{w}_{1} \\
\widetilde{w}_{2} \\
\widetilde{w}_{3} \\
\widetilde{z}_{1} \\
\widetilde{z}_{2}
\end{array}\right)=\left(\begin{array}{c}
w_{1} \cosh (x \sinh c)+z_{1} \sinh (x \sinh c) \\
w_{2} \cos (y \cosh c)-w_{3} \cosh c \sin (y \cosh c) \\
w_{3} \cos (y \cosh c)+w_{2} \operatorname{sech} c \sin (y \cosh c) \\
z_{1} \cosh (x \sinh c)+w_{1} \sinh (x \sinh c) \\
w_{3} \sinh c \cos (y \cosh c)+w_{2} \tanh c \sin (y \cosh c)
\end{array}\right) .
$$

The Ribaucour transformation for the degenerate surface $X_{1}=(x, 0,0)$ is

$$
\widetilde{X}_{1}=\left(\begin{array}{l}
x \\
0 \\
0
\end{array}\right)+r\left(\begin{array}{c}
\sinh (x \sinh c) \\
\cos y \cos (y \cosh c)+\operatorname{sech} c \sin y \sin (y \cosh c) \\
-\sin y \cos (y \cosh c)+\operatorname{sech} c \cos y \sin (y \cosh c)
\end{array}\right),
$$

where

$$
r=\frac{-2 \cosh (x \sinh c)}{\sinh c\left\{\cosh ^{2}(x \sinh c)-\tanh ^{2} c \sin ^{2}(y \cosh c)\right\}} .
$$

The new solution $\widetilde{\phi}$ of the sinh-Gordon equation is

$$
\widetilde{\phi}=\log \frac{\cosh (x \sinh c)-\tanh c \sin (y \cosh c)}{\cosh (x \sinh c)+\tanh c \sin (y \cosh c)} .
$$
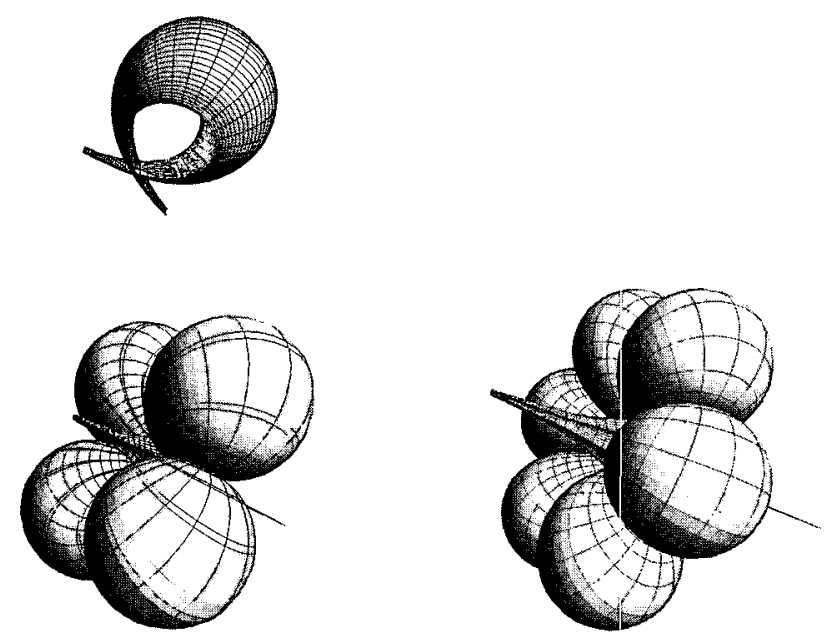
The above pictures are the immersion $\widetilde{X}_{1}(0<y<\pi / 2)$, and the full images of $\widetilde{X}_{1}$ for $\cosh c=2$ and $\cosh c=1.5$, respectively.

\section{References}

[1] L. Bianchi, Vorlesungen über Differentialgeometrie, Leipzig, Berlin, Druck und Verlag von B. G. Teubner (1910).

[2] M. Brück, X. Du, J. Park, and C. L. Terng, The submanifold geometry of real Grassmannian systems, Mem. Amer. Math. Soc. 155 (2002), No. 735.

[3] F. Burtall, Isothermic surfaces: conformal geometry, Clifford algebras and integrable systems, Integrable systems, Geometry and Topology, International Press, to appear.

[4] J. Inoguch, Characterizations of Bäcklund transformations of constant mean curvature surfaces, International Jour. Math. 16 (2005), 101-110.

[5] U. Hertrich-Jeromin and F. Pedit, Remarks on the Darboux transform of isothermic surfaces, Doc. Math. 2 (1997), 313-333.

[6] J. Park, Riemannian submanifolds in Lorentzian manifolds with the same constant curvatures, Bull. Korean Math. Soc. 39 (2002), no. 2, 237-249.

[7] _ Lorentzian submanifolds in Lorentzian space form with the same constant curvatures, Geom. Ded. 108 (2004), 93-104.

[8] C. L. Terng, Soliton equations and differential geometry, Jour. Diff. Geom. 45 (1997), 407-445.

Department of Mathematics

Dongguk University

Seoul 100-715, Korea

E-mail: jpark@dgu.edu 